\title{
ACCESSING REMOTE APPLICATION WITHOUT INSTALLATION AT HOST END THROUGH CLOUD COMPUTING
}

\author{
Shaily malik \\ Lecturer, CSE Dept. \\ Maharaja Surajmal Instt. Of Technology \\ Janakpuri, New Delhi-110058
}

\author{
Vineet Sinha \\ B.Tech (Dept of CSE) $3^{\text {rd }}$ year Student \\ Maharaja Surajmal Instt. Of Technology \\ Janakpuri , New Delhi-110058
}

\begin{abstract}
Cloud computing is an upcoming efficient networking computing environment. Our work proposes sharing an application across various clients of a host system via cloud computing. The sharing is performed between the host storage system and the remote storage system in a cloud computing environment. The Host storage device of the host system is updated to retrieve the application from the remote storage device that is shared through cloud computing environment. The application now can be accessed by the host processing devices. The application can be implemented on the host processing device without requiring any installation of the application on the host processing device. A bunch of function calls are performed between the operating system of the host processing device and the application residing on the host storage device. The system has a cloud interface between remote and host machines to update the host storage device about any changes in the application at the remote storage device. This leads to the better utilization of the cloud bandwidth and the host resources such as storage, processors etc.
\end{abstract}

\section{Categories and Subject Descriptors} H.3 [Information Storage and Retrieval]: Systems and Software-Distributed Systems; C.2 [Computer Communication Networks] : Distributed Systems Network Operating Systems.

\section{General Terms}

Computing theory

\section{Keywords}

Cloud Computing, remote storage, host machine

\section{INTRODUCTION}

This topic has recently gained significant momentum and attention in both academia and corporate world. It's generally accepted that cloud computing is a hot and new IT paradigm for users.

The main idea is to make applications available on flexible execution environments primarily located in the Internet. Infrastructure as a service refers to the sharing of hardware resources for executing services[1].

In short, cloud computing has become a significant technology trend and could reshape the IT sector and the IT marketplace.

\section{CLOUD COMPUTING}

\subsection{What is Cloud Computing?}

Cloud computing provides a platform to use the shared computing resources[4]. It groups large number of computing servers and other resources and typically offers their combined capacity on an on- demand, pay per cycle basis. Cloud Computing refers to both the applications delivered as services over the Internet and the hardware and systems software in the datacenters that provide those services. End users can access the service "anytime, 
anywhere", share data and collaborate more easily, and keep their data stored safely in the infrastructure. When a Cloud is made available in a pay-as-you-go manner to the public, we call it a Public Cloud; the service being sold is Utility Computing[5].

\subsection{Why Cloud Computing?}

If a user interacts with any online application, email, online social networking, online slide show creation, online design tools, online mind mapping and file conversion, collaborative software, social media, online data storage or any other internetbased application, actually user interacts with Cloud Computing environment[5].

Some applications are easy to use and others are waiting to get in touch with cloud computing.

\section{CLOUD COMPONENTS}

The cloud computing network consists of communicating cloud components such as WAN, remote storage, host storage. The cloud network is communicatively coupled with WAN, remote storage and host storage system. The host storage system includes multiple host processing devices communicating via LAN by using network switches and/or routers, wireless routers, DSL, cable modem. Data that is to be communicating between remote and host systems travel across a WAN and enter into the network cloud where it is connected with other data transmissions[2]. The WAN uses circuit switching and/or packet switching methods for enabling communication. The standard network protocols such as TCP/IP and/or Remote Desktop Protocol (RDP) are used to perform deliver transport and addressing functions[3].

The cloud computing network consists of multiple storages which are clustered and partitioned into primary and archive storages. The archive storage becomes functional whenever primary storage suffers from any failure and unable to serve client access request. The clusters are associated with network switches/routers to enable communication among different parts of clusters.

\section{ACCESS WITHOUT INSTALLATION}

As illustrated in fig.1 an application installed on a remote storage can be accessed through cloud interface and passes it to the host storage of the host system. The two locators operational between remote storage and host storage as well as host system respectively. The two locators work in similar fashion and interact mutually in order to communicate appropriate application information stored in various locations of the system.

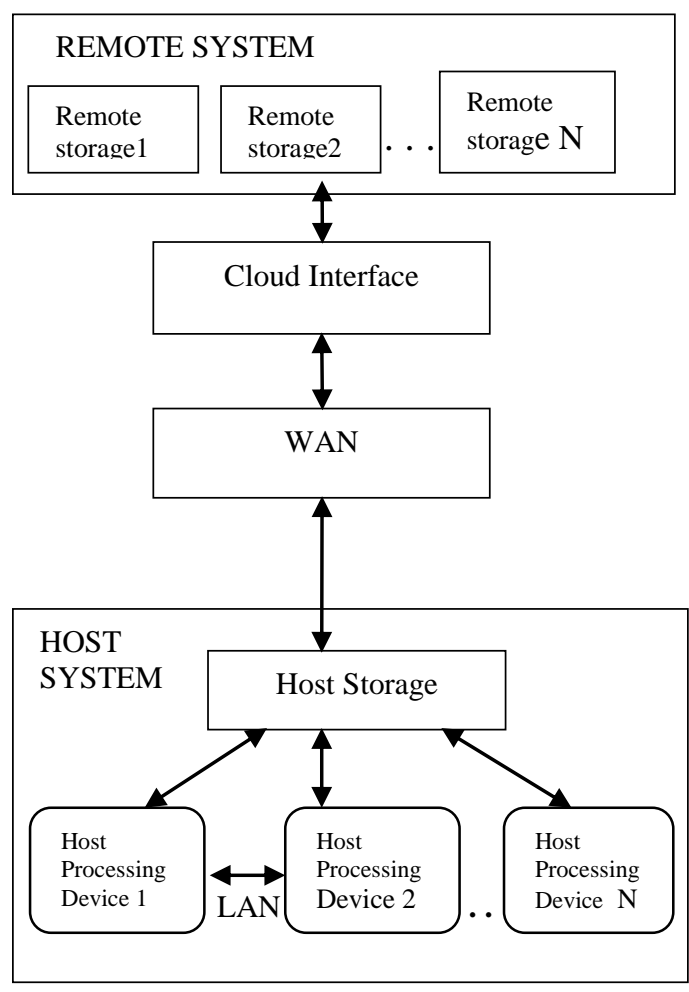

Fig.1 Accessing without Installation

\section{EXECUTION MANAGER}

The application execution manager of the host system implements the application on a host 
processing device without installation in the host storage device. The execution manager retrieves and accesses the application from a storage device if:

(a).The requested application do not exist with storage device, and

(b).WAN bandwidth is higher than LAN bandwidth.

The system includes host storage in the host system. The host storage and the other processing devices are communicatively coupled through the network to access the application from the cloud computing platform and store the application on a memory of the host storage[6]. The host processing devices accesses the application from the host storage and runs the application without installing the application on the processing devices.

Further, the execution manager consists of examining module and host system configuration manager which is communicatively associated with the host storage device and the host processing device with LAN and transports the data independent of a WAN associated with the host storage device in the cloud computing platform.

\section{UPDATING MANAGER}

The host system includes the host cache which stores the data set output from running the application on the host processing device. The stored data set of application is couple with LAN and it is periodically updated by "Updating Manager". Updating manager illustrated in fig.1 updates the host storage device and gets the application from a remote storage device of a cloud computing system. Whenever the application is required, the host processing device accesses the application from the host storage without the application at the host storage device[7]. The without installation on a host processing device signifies that the operating system is not modified. The application at host processing device can be deleted after the implementation to avoid any data duplication or unauthorized access[4].

\section{CONCLUSION}

Cloud computing is an emerging computing paradigm for the IT leaders. The corporate giants such as Google, Dell, HP, Sun Microsystems, Yahoo and IBM are accelerating cloud computing. However, the discussed research facts in cloud computing are still inadequate and waiting for more efforts to repaint the IT sector.

In this paper, we have drawn up improvement of network bandwidth utilization in cloud computing. This paper models cloud computing as a better and effective place for optimal usage of computer resources in LAN and WAN connectivity. The paper primarily promotes the accessing of remote application at client end without installing the application at client end.

\section{REFERENCES:}

[1] R. Buyya, Rajiv Ranjan, and Rodrigo N. Calheiros. Modeling and Simulation of Scalable Cloud Computing Environments And the CloudSim Toolkit: Challenges and Opportunities. Proceedings of the 7th High Performance Computing and Simulation (HPCS 2009) Conference, Leipzig, Germany,June 21 - 24, 2009.

[2] Börje Ohlman, Anders Eriksson and René Rembarz. What Networking of Information Can Do for Cloud Computing. Proceedings of 18th IEEE International Workshops on Enabling Technologies: Infrastructures for Collaborative Enterprises,2009,pp.78-83.

[3] Demers, A. J., Petersen, K., Spitzer, M. J., Terry, D. B., Theimer, M. M., and Welch, B. B. The bayou architecture: Support for data sharing among mobile users. In Proceedings IEEE Workshop on Mobile Computing Systems \& Applications (Santa Cruz, California, August-September 1994), pp. 27.

[4] Lijun Mei,W.K. Chang, T.H. Tse. A Tale of Clouds: Paradigm Comparisons and Some Thoughts on Research Issues. Proceedings of the 2008 IEEE Asia-Pacific Services 
Computing Conference (APSCC 2008), IEEE Computer Society Press, Los Alamitos, CA (2008).

[5] Mr. P. Boominathan, S.AjayKumar, C.Nachiappan, K.Periyakaruppan. Enhancing Portable Environment using Cloud and Grid. Proceedings of the 2009 International conference on Computer Design and Applications(ICCSIT 2008), Singapore(2008).
[6] E. Hand. Head in the clouds, Nature, (449):963, Oct 2007.

[7] Jeremy Geelan. Twenty one experts define cloud computing. Virtualization, August 2008. Electronic Magazine, article available at http://virtualization.syscon.com/node/612375. 